\title{
Optode Design Space Exploration for Clinically-robust Non-invasive Fetal Oximetry
}

\author{
DANIEL D. FONG, VIVEK J. SRINIVASAN, KOUROSH VALI, and SOHEIL GHIASI, \\ University of California, Davis
}

\begin{abstract}
Non-invasive transabdominal fetal oximetry (TFO) has the potential to improve delivery outcomes by providing physicians with an objective metric of fetal well-being during labor. Fundamentally, the technology is based on sending light through the maternal abdomen to investigate deep fetal tissue, followed by detection and processing of the light that returns (via scattering) to the outside of the maternal abdomen. The placement of the photodetector in relation to the light source critically impacts TFO system performance, including its operational robustness in the face of fetal depth variation. However, anatomical differences between pregnant women cause the fetal depths to vary drastically, which further complicates the optical probe (optode) design optimization. In this paper, we present a methodology to solve this problem. We frame optode design space exploration as a multi-objective optimization problem, where hardware complexity (cost) and performance across a wider patient population (robustness) form competing objectives. We propose a model-based approach to characterize the Pareto-optimal points in the optode design space, through which a specific design is selected. Experimental evaluation via simulation and in vivo measurement on pregnant sheep support the efficacy of our approach.
\end{abstract}

CCS Concepts: • Applied computing $\rightarrow$ Life and medical sciences; $\bullet$ Computer systems organization $\rightarrow$ Embedded and cyber-physical systems; Dependable and fault-tolerant systems and networks;

Additional Key Words and Phrases: Internet of medical things, Non-invasive fetal oximetry, medical cyberphysical systems, design optimization, design space exploration, multi-objective optimization

\section{ACM Reference format:}

Daniel D. Fong, Vivek J. Srinivasan, Kourosh Vali, and Soheil Ghiasi. 2019. Optode Design Space Exploration for Clinically-robust Non-invasive Fetal Oximetry. ACM Trans. Embed. Comput. Syst. 18, 5s, Article 63 (October 2019), 22 pages.

https://doi.org/10.1145/3358207

\section{INTRODUCTION}

The current technique for monitoring fetal well-being during labor is to use cardiotocography, which analyzes the temporal relationship between the fetal heart rate and uterine contractions. Unfortunately, this approach was adopted without the support of a clinical trial and is plagued with a high-rate of false positives ( $99.8 \%$ for cerebral palsy) [20]. This has led to a significant increase in emergency $\mathrm{C}$-sections, while the rates of adverse delivery outcomes have remained the same [1].

This article appears as part of the ESWEEK-TECS special issue and was presented in the International Conference on Hardware/Software Codesign and System Synthesis (CODES+ISSS), 2019.

Authors' address: D. D. Fong, V. J. Srinivasan, K. Vali, and S. Ghiasi, University of California, Davis, 1 Shields Avenue, Davis, CA, 95616; emails: \{dfong, vjsriniv, kvali, ghiasi\}@ucdavis.edu.

Permission to make digital or hard copies of all or part of this work for personal or classroom use is granted without fee provided that copies are not made or distributed for profit or commercial advantage and that copies bear this notice and the full citation on the first page. Copyrights for components of this work owned by others than ACM must be honored. Abstracting with credit is permitted. To copy otherwise, or republish, to post on servers or to redistribute to lists, requires prior specific permission and/or a fee. Request permissions from permissions@acm.org.

(c) 2019 Association for Computing Machinery.

1539-9087/2019/10-ART63 \$15.00

https://doi.org/10.1145/3358207 

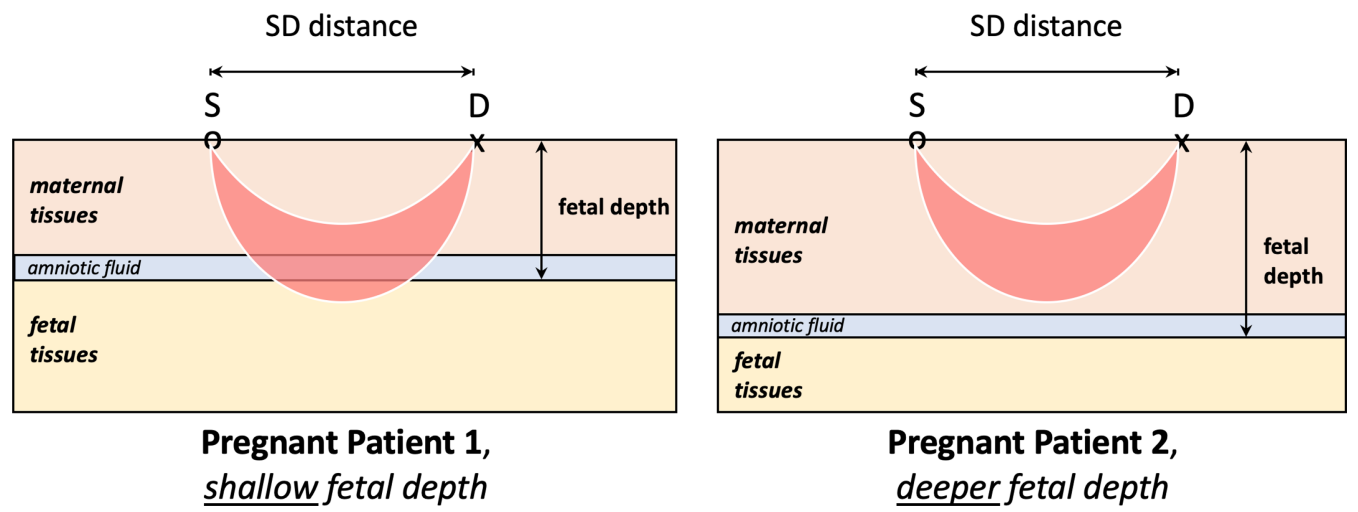

Fig. 1. Patient variability issues related to variations in fetal depth between two pregnant women. Light captured by the optode on Patient 2, represented by the red arc between the light source (S) and photodetector (D), does not traverse fetal tissues and causes the TFO performance to suffer.

In the United States, 1 of every 3 births result in a C-section [19], which is a major abdominal surgery and can increase health risks to both mother and child, such as higher rates of chronic lung conditions, type-1 diabetes, and other post-operative complications [6, 14, 21]. Notably, a large proportion of these surgeries are performed in response to cardiotocography traces [2], making it clear that this high-cost (increased C-sections) and low-benefit (no reduction in rates of harm) fetal monitoring technique needs an alternative.

We have been working on the development of a non-invasive transabdominal fetal pulse oximeter (TFO) to provide intrapartum ${ }^{1}$ fetal oxygenation and help physicians make well-informed decisions regarding an emergency $\mathrm{C}$-section. It does this by sending photons towards the fetus through the maternal abdomen, and analyzing the changes in light intensity (signal) caused by fetal tissues using an optical probe (optode). For reflectance-mode optodes, the placement of a detector relative to the light source (source-to-detector or SD distance) helps to capture photons that traverse a particular depth of tissue. To probe shallow tissues, a small SD distance is sufficient. Deeper tissues can be investigated by using a larger SD distance, but at the expense of capturing fewer photons overall (decreased light intensity). As such, tuning this parameter can improve the quality of the signal to investigate deep fetal tissue. However, anatomical differences between pregnant women can cause the fetal depths to vary drastically, making the optimal SD separation different for each patient, as illustrated in Figure 1. In fact, a preliminary study saw that differences in the fetal depth between pregnant women had a large effect on the signal-to-noise ratio [36] which highlights that challenges regarding patient variability must be addressed before clinical adoption.

Clinically-robust systems must be able to deliver sufficient performance across the wide-range of patient variability, but often increase its design complexity. We suggest that designing such a system in a cost-effective manner can be solved by framing it as a multi-objective optimization problem, where each patient wants the maximum system performance for their particular physical characteristics (robustness) and a designer wants to minimize the hardware complexity (cost) of the system. In this paper, we propose a methodology that solves this problem by considering the overall performance across the patient-population. We use this method to optimize the optode design for TFO, towards developing a clinically-robust non-invasive fetal oximetry system that addresses patient variability. Our contributions in this paper are the following:

${ }^{1}$ Occurring during labor and delivery. 
- We perform a Design Space Exploration (DSE) to characterize the system performance with respect to patient variability and different optode designs.

- We propose a population-conscious approach to select a preferred Pareto-optimal optode design that minimizes the number of detectors and maximizes the performance across the population.

- We develop a TFO system prototype and present preliminary results captured on pregnant sheep to highlight the in vivo feasibility to capture the fetal signal.

\section{BACKGROUND}

\subsection{Multi-objective Optimization Problems}

Multi-objective optimization problems (MOOP) are often presented in the context of needing to make decisions based on a number of different criteria, where the goal is to identify one (or a set of) feasible solutions that provide the best tradeoffs between objectives. The general form of a multiobjective optimization problem is expressed as the joint optimization ${ }^{2}$ of $n$ objective functions, where the goal is to identify an optimal solution vector $x$ that expresses the particular assignment of $m$ decision variables and exists in the feasible solution set $X$.

$$
\begin{array}{cl}
\text { Min } & f(x)=\left(f_{1}(x), f_{2}(x), \ldots, f_{n}(x)\right) \\
\text { s.t. } & x=\left(\alpha_{1}, \alpha_{2}, \ldots, \alpha_{m}\right) \in X
\end{array}
$$

2.1.1 Pareto Optimality. Given the potential for conflicting objectives, a single optimal solution may not exist and thus tradeoffs must be considered. Since objectives are often not directly comparable with each other, solutions are ranked using a dominance relationship. In particular, a solution $x_{1} \in X$ is said to dominate another solution $x_{2} \in X$ if two conditions are met:

(1) $x_{1}$ is at least as good as $x_{2}$ for all objectives.

(2) $x_{1}$ is strictly better than $x_{2}$ in at least one objective.

The set of non-dominated solutions is called the Pareto-optimal set (or Pareto frontier) and represents the solutions that provide the best tradeoffs (e.g. improving $f_{1}(x)$ results in degrading $f_{2}(x)$ ). Since it can be difficult to faithfully represent the ranking importance of various objectives, the Pareto frontier is often presented to a designer who evaluates the tradeoffs and selects a preferred solution. Thus, generating (or approximating) a set of diverse Pareto-optimal solutions is often the goal of multi-objective optimization problems.

Design Space Explorations (DSE) are often used to generate a mapping between the objective and solution spaces, which is used to check for dominance relationships. Afterwards, the solutions are analyzed and filtered for Pareto-optimality before being presented to the designer. However, sometimes a designer wants a single Pareto-optimal solution to be suggested. In these cases, scalarization techniques can be a useful tool. These approaches combine (or reduce) objectives by incorporating some of the designer's preferences to create a single-objective to optimize. Two commonly-used techniques are the Weighted-Sums and $\varepsilon$-Constraint Methods.

2.1.2 Weighted-Sums Method. As the name implies, this technique combines multiple objectives by taking the weighted sum of each objective's product with a user-specified weighting factor [32].

$$
\begin{array}{ll}
\text { Min } & f(x)=\sum_{i=1}^{n} w_{i} * f_{i}(x) \\
\text { s.t. } & x \in X
\end{array}
$$

\footnotetext{
${ }^{2}$ Multiplying an objective function by -1 allows us to perform a maximization procedure, without losing the general minimization semantics.
} 
While this approach is intuitive and simple to calculate, it can be difficult to select appropriate weights that faithfully represent the relative importance of each objective in a desired region of the solution space. This is evident when the units of each objective are not matched or are incapable of being quantified. In these cases, ambiguous values may be assigned which can lead to undesired solutions being presented.

2.1.3 $\varepsilon$-Constraint Method. In this approach, a single most-important objective is identified and retained for optimization, while the others are turned into constraints with user-specified bounds [13]. Solutions meeting those constraints are then solved for in the single-objective case and presented as a Pareto-optimal point.

$$
\begin{array}{cl}
\text { Min } & f_{i}(x) \\
\text { s.t. } & \varepsilon_{k}^{L} \leq f_{k}(x) \leq \varepsilon_{k}^{U} \\
& x \in X \\
& k=\{1, \ldots, K\} \text { and } k \neq i
\end{array}
$$

Similarly, it can be difficult to find appropriate values for the lower- and upper- bounds $\left(\varepsilon_{k}^{L}\right.$ and $\left.\varepsilon_{k}^{U}\right)$ such that feasible solutions exist. This can be solved by finding the feasible range of each $f_{k}(x)$ first, and then applying appropriate bounds in an a posteriori manner. As such, these bounds can be iteratively tightened, relaxed, or moved to identify solutions at different points in the feasible solution space, at the cost of redundant calculations.

\subsection{Overview of Pulse Oximetry}

Pulse oximetry non-invasively estimates the relative amount of oxygenated hemoglobin in the blood (arterial oxygen saturation or $\mathrm{SpO}_{2}$ ) by analyzing the changes in light intensity (of at least two wavelengths) caused by pulsating arterial tissues. At its core, this is described by the BeerLambert Law $[3,18]$ which relates the exponential decay of incident light intensity entering a medium $\left(I_{0}\right)$ to the light intensity reaching a detector $\left(I_{d e t}\right)$ using the optical properties and composition of the medium $\left(\mu_{a}\right)$ and the path-length photons take to get to the detector $(L)$.

$$
I_{\text {det }}=I_{0} * e^{\left(-\mu_{a} * L\right)}
$$

This can be rewritten in terms of temporal changes in light absorption $(\triangle A)$ and expanded to incorporate multiple tissues $(i)$ with the Modified Beer-Lambert Law [8]:

$$
\Delta A(\lambda)=-\ln \left(I_{t 1} / I_{t 0}\right)=\sum_{i} \Delta \mu_{a, i}(\lambda) *\left\langle L_{i}\right\rangle
$$

where $I_{t 0}$ is the measured light intensity, of wavelength $\lambda$, captured at time $t 0$, and $I_{t 1}$ is the light intensity at a later time $t 1$. The expected path-length $\left(\left\langle L_{i}\right\rangle\right)$ is used here since discontinuities in the refractive index between and within tissues cause photons to scatter and take a tortuous path to the detector.

Since oxy- and deoxy- hemoglobin $\left(\mathrm{HbO}_{2}\right.$ and $\left.\mathrm{Hb}\right)$ absorb light at different levels in the red and near-infrared spectrums, the relative light attenuation seen at these wavelengths changes depending on how oxygenated the blood is. This is important for pulse oximetry, where the oxygen saturation $\left(\mathrm{SpO}_{2}=\left[\mathrm{HbO}_{2}\right] /\left(\left[\mathrm{HbO}_{2}\right]+[\mathrm{Hb}]\right)\right)$ is estimated using the modulation ratio $\mathrm{R}$, which is a ratio of the changes in light absorption $(\Delta A)$ from pulsating arterial tissues ${ }^{3}\left(\Delta \mu_{a}\right)$ at two

\footnotetext{
${ }^{3}$ Each heart contraction changes the arterial blood volume at systole and diastole, which results in temporal changes in light intensity.
} 


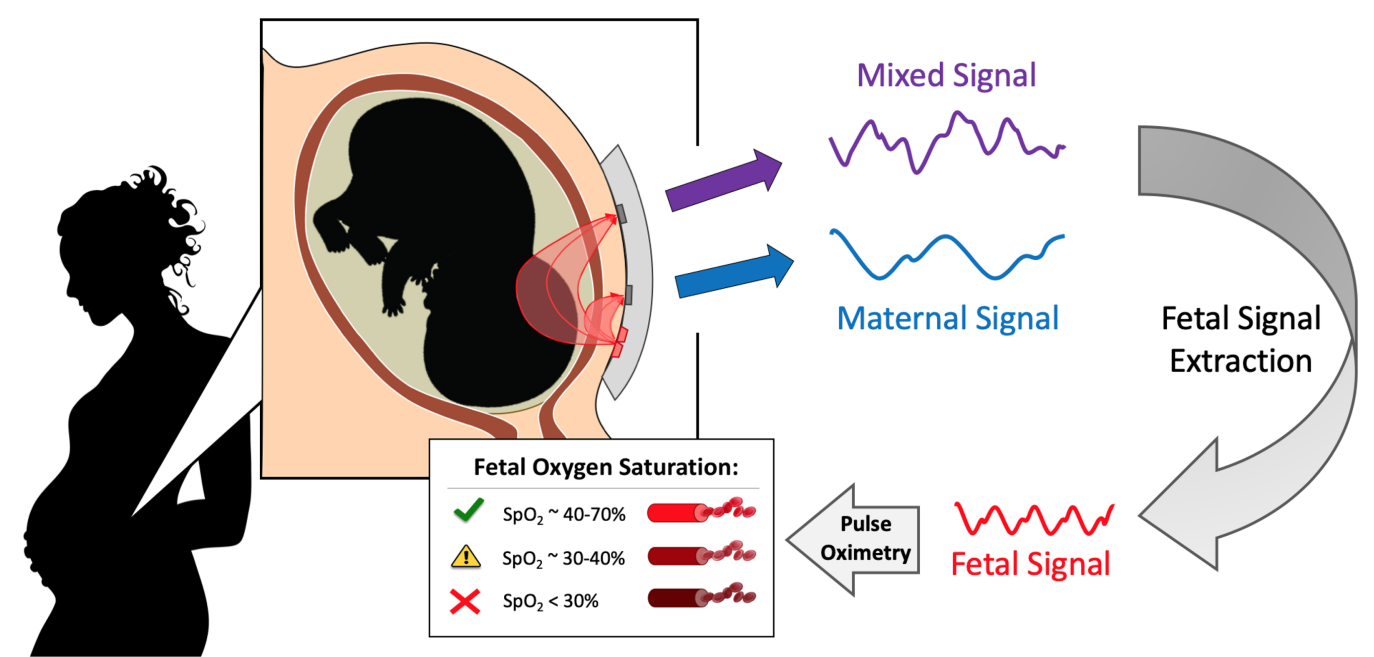

Fig. 2. High-level diagram of our non-invasive, Transabdominal Fetal Pulse Oximetry (TFO) system. Some of the light captured at the photodetectors carries fetal information and can be used to estimate the fetal oxygen saturation.

wavelengths and is proportional to $\mathrm{SpO}_{2}[31]$.

$$
R=\frac{\Delta A_{\lambda_{1}}}{\Delta A_{\lambda_{2}}}=\frac{\ln \left(I_{\text {systole }, \lambda_{1}} / I_{\text {diastole }, \lambda_{1}}\right)}{\ln \left(I_{\text {systole }, \lambda_{2}} / I_{\text {diastole, } \lambda_{2}}\right)}=\frac{\Delta \mu_{a, \lambda_{1}}\langle\measuredangle\rangle}{\Delta \mu_{a, \lambda_{2}}\langle\measuredangle\rangle} \propto S p O_{2}
$$

Calibrated pulse oximeters find the wavelength-specific proportionality factor between $\mathrm{R}$ and $\mathrm{SpO}_{2}$ using invasive blood-gas analysis measurements on a large number of healthy volunteers in controlled desaturation experiments.

\subsection{Transabdominal Fetal Pulse Oximetry}

Transabdominal fetal pulse oximetry (TFO) is a fully non-invasive technique to estimate the fetal oxygen saturation $\left(\mathrm{SpO}_{2}\right)$ by using light to investigate the fetal tissues with a reflectance-mode optical probe (optode), and is illustrated in Figure 2. This is performed by sending photons towards the fetus at the maternal abdomen using an emitter. As photons propagate through the tissue, some of them are extinguished (via absorption) or scattered from their initial trajectory, increasing their path within the tissue and allowing it to diffuse towards deeper tissues. Some of the light that reaches the fetus scatters towards the skin surface and is captured by a photodetector as a mixed (maternal+fetal) signal. This mixed signal is then processed to extract the fetal contribution and analyzed to estimate fetal $\mathrm{SpO}_{2}$ through conventional pulse oximetry. This involves identifying the fetal photo-plethysmogram (PPG) at two wavelengths, which is characterized by small, repeated dips in light intensity occurring at the fetal heart rate. In contrast, changes in light intensity due to maternal tissues (noise) are much stronger and causes the fetal signal-to-noise ratio to be inadequate unless the maternal noise is filtered from the mixed signal. Importantly, fetal heart rates $(\sim 110$ $240 \mathrm{bpm})$ are normally higher than the maternal heart rates $(\sim 60-100 \mathrm{bpm})$ and allow a number of different approaches to improve the fetal signal-to-noise ratio, and are discussed in Section 2.3.2.

2.3.1 Patient Variability. Both anatomical and physiological differences between and within patients can have large effects on the ability to capture the fetal signal using TFO. For example, natural variations in the fetal depth change the distance photons must travel to reach the fetal tissue, which highly affects the amount of fetal information captured at the photodetector and 
thus system performance. This can be caused by differences in the overall thickness of abdominal tissue, spontaneous changes in fetal location, or through the progression of natural birth via labor dynamics. In addition, the mother's physiological systems can cause temporal changes in the optical properties of superficial tissue, which can further complicate investigating the deep fetal tissue. For example, the maternal heart contractions cause the blood-tissue volume in the superficial tissue to increase slightly and causes the signal to reflect maternal characteristics. This systemic noise makes it harder to distinguish the changes caused by deeper fetal tissues (i.e. fetal signal) and requires a processing technique that can adapt to changes in physiology. Designing a system that can account for both inter- and intra- patient variability is critical to the development of a clinically-robust device.

2.3.2 Fetal Signal Extraction. To investigate the fetal tissue using TFO measurements, we must first extract the fetal signal from the mixed signal measurement. To do this, we utilize a pair of detectors at different SD distances (one near the source and another far away) to capture the relative difference of maternal-fetal influence at each detector, which is used to remove the maternal noise seen in the mixed signal. To illustrate how this works, Equations (5) and (6) describe the absorption seen at the near and far detectors respectively,

$$
\begin{aligned}
\Delta A_{N} & =\Delta \mu_{a, \text { mat }} *\left\langle L_{\text {mat }}\right\rangle_{N}+\Delta \mu_{a, f e t} *\left\langle L_{f e t}\right\rangle_{N} \\
\Delta A_{F} & =\Delta \mu_{a, \text { mat }} *\left\langle L_{\text {mat }}\right\rangle_{F}+\Delta \mu_{a, f e t} *\left\langle L_{f e t}\right\rangle_{F}
\end{aligned}
$$

where subscripts $N$ and $F$ correspond to the near and far detectors, and $\left\langle L_{\text {mat }}\right\rangle$ and $\left\langle L_{f e t}\right\rangle$ represents the expected partial path-lengths a photon took through the maternal and fetal tissues, respectively, to reach the detectors. When the near detector is sufficiently close to the source, most of the photons it captures will have only traversed maternal tissues, namely $\left\langle L_{f e t}\right\rangle_{N} \approx 0$. By utilizing this difference in maternal-fetal information at the near-far detectors, we can extract the fetal signal $\left(\triangle A_{f e t}\right)$ by:

$$
\Delta A_{f e t}=\Delta \mu_{a, f e t} *\left\langle L_{f e t}\right\rangle_{F}=\Delta A_{F}-\alpha * \Delta A_{N}
$$

where $\alpha$ is a scaling factor that represents a difference in the partial path-lengths through the maternal tissue seen at the two detectors, $\alpha=\left\langle L_{m a t}\right\rangle_{F} /\left\langle L_{m a t}\right\rangle_{N}$, which can be different for each patient and is generally unknown [25, 34]. To account for this, we utilize adaptive noise cancellation (ANC) techniques to implement Equation (7) and find $\Delta A_{\text {fet }}$, where the amount of noise to be removed from a noisy signal $\left(\Delta A_{F}\right)$ is estimated using a noise reference $\left(\Delta A_{N}\right)$ and an adaptive filter $(\alpha)$ that is tuned by minimizing the error in an optimization parameter. The extracted fetal signal, captured at two wavelengths, is then used with Equation (4) to estimate fetal $\mathrm{SpO}_{2}$ as in conventional pulse oximetry calculations. Studies have shown that ANC is superior at extracting the fetal signal $[10,33]$. However, its performance in relation to the differences in fetal depth remains unexplored and is investigated in this paper.

\section{MOTIVATION AND PROBLEM STATEMENT}

\subsection{Addressing Patient Variability}

As described earlier, an optimal optode design must strike a balance between signal strength and sensitivity for a targeted tissue depth, but fetal depth variations (patient variability) can cause the optimal design to be different between patients. Since patient characteristics are outside the realm of control, improvements to the optode design must be made.

An effective-but-costly solution is to improve the optode's dynamic range by increasing the number of detectors and varying their SD distances to improve the range of fetal depths captured 
by the system. In an unlimited-resources scenario, an optode containing a large number of detectors would capture photons across the entire maternal abdomen to maximize the range of fetal depths investigated. This approach is impractical from a clinical viewpoint, has diminished returns since many detectors will capture redundant or unhelpful information to extract the fetal signal, and can drastically increase development time and costs. On the other hand, a resource-limited approach runs the risk of using an insufficient number of detectors, resulting in sub-optimal performance across the population. Realistically, a balance must be made between these two approaches.

\subsection{Problem Statement}

A clinically-robust, non-invasive fetal oximetry system must be able to provide sufficient performance across the wide-range of patient variability, in which the optode design plays a critical role. However, designing an optode that both maximizes the performance across the population and minimizes hardware complexity requires careful evaluation of the tradeoffs between designs. In this paper, we seek to answer the following question:

"What are the optode design parameters that maximize TFO system performance across the patient population and minimize the number of detectors on the optode?"

\section{PROPOSED APPROACH}

We suggest that this question can be addressed by approaching it as a multi-objective optimization problem, where our competing objectives are to maximize the performance for each patient and to minimize the number of components in the system. To identify Pareto-optimal solutions, the system performance for each patient characteristic is compared over different optode designs. However, outliers in patient characteristics might lead to designs that are less beneficial for more common situations. To address this, we propose a population-conscious methodology which identifies a Pareto-optimal solution that maximizes the potential benefit for a majority of the patients while minimizing the number of additional components. It does this by optimizing for a population-based performance metric and turning the number of components into a user-specified constraint.

Given the broad nature of patient variability and the many design choices involved with developing a TFO system, we focus this investigation on several key parameters, which are described and defined below:

System parameters, $\mathrm{x}$ : represents a potential solution or particular assignment of design parameters. Since the optode plays a crucial role in addressing patient variability, we focus on parameters directly related to improving the optode design, namely the number of detectors and their $S D$ distances. As previously mentioned, increasing the number of detectors and varying their SD distance improves the dynamic range of fetal depths an optode can achieve by capturing photons that traverse a wider variety of paths through the underlying tissue. Other system parameters, were assigned fixed values to focus the scope of investigation and improve computational feasibility.

Patient characteristics, $\mathrm{p}_{\mathrm{i}}$ : defines the particular characteristics of the $i^{\text {th }}$ patient in the population $P_{p o p}$. Given the broad nature of characteristics that can cause measurement variability, we focus on the fetal depth and maternal heart rate seen in near-term pregnant women. Intuitively, increased fetal depth has an adverse effect on the ability to detect the fetal signal, since light intensity decays with increased distance photons must travel, as described by Equation (2).

System performance, $\mathrm{s}_{\mathrm{i}}(\mathrm{x})=s\left(p_{i}, x\right)$ : describes the system performance on the $i^{\text {th }}$ patient using a system with design parameters $x$. In the case of TFO, we define system performance as the maximum fetal signal-to-noise ratio (SNR) captured on an optode and use 
it interchangeably with the term 'performance' throughout this paper. We use the maximum SNR as an objective measure to compare solutions since optodes can have a different number of near-far detector combinations.

Hardware complexity, $\mathrm{k}(\mathrm{x})$ : is the function that describes the hardware complexity (costs) for the system. We let $k(x)$ express the number of detectors on the optode $x$, since it directly relates to increased resource-usage and development effort (complexity of designing an embedded system that interacts with each of the detectors).

Using this notation, our overarching goal is to optimize the set of objective functions $\left(s_{i}(x)\right.$ and $k(x)$ ), to identify the optode design parameters $(x)$ that addresses issues related to patient variability amongst near-term pregnant women $\left(P_{p o p}\right)$ :

$$
\begin{array}{cl}
\text { Min } & \left(k(x),-s_{1}(x),-s_{2}(x), \ldots,-s_{N}(x)\right) \\
\text { s.t. } & x \in X \text { and } p_{i} \in P_{p o p} \text { for } i=1,2, \ldots, N
\end{array}
$$

where $N$ is the number of different patient characteristics to account for in the population set $P_{\text {pop }}$ and $X$ is the set of feasible solutions.

Using the proposed method, we simplify this ' $N+1$ ' -objective optimization problem by defining a single population-performance metric $\left(S_{\text {pop }}(x)\right)$ to optimize and turn $k(x)$ into a constraint.

$$
\begin{array}{cl}
\operatorname{Max} & S_{p o p}(x)=\sum_{i} w_{i} * s_{i}(x) \\
\text { s.t. } & k(x) \leq \varepsilon \\
& x \in X \\
& i \in\{1,2, \ldots, N\}
\end{array}
$$

To create the population-performance metric $\left(S_{p o p}(x)\right)$, we must provide patient-specific weights $\left(w_{i}\right)$ that reflect the relative incidence of particular characteristics $\left(p_{i}\right)$. We do this by using the distribution of $p_{i}$ across $P_{p o p}$ as the weighting function, which provides an objective measure of the relative importance between designing a system for common- vs uncommon- cases. Afterwards, Pareto-optimal solutions are found by setting an upper-bound $\varepsilon$ on the number of detectors potential optodes can have $(k(x))$ and optimizing for $S_{\text {pop }}(x)$. To select a preferred Pareto-optimal design, we vary the bounds of $k(x)$ and identify the solution that provides the best return-oninvestment, namely identifying the point at which performance-benefits taper-off for each additional detector (diminishing returns).

4.0.1 Discretization/Clustering. Evaluating the system performance $s_{i}(x)$ for a given solution $x$ and patient characteristic $p_{i}$ can be computationally expensive. Since similar patient characteristics and system parameters often result in similar performance, quantizing the exploration parameters into discrete values can help improve the computational efficiency while still providing useful solutions. In addition, heuristic-based searched methods can also be used to improve the efficiency at which the solution space is explored when optimizing parameters [17].

\section{CASE STUDY: OPTODE DESIGN OPTIMIZATION FOR TFO}

To highlight its efficacy, we use the proposed approach to optimize the optode design for noninvasive fetal oximetry and present it as a case study. To do this, we perform an optode design space exploration to evaluate the relationships that optode design $(x)$ and patient characteristics $\left(p_{i}\right)$ have on system performance $\left(s\left(p_{i}, x\right)\right)$. We take a model-based approach to quantify the system 
Table 1. Dimensions of Patient Variability Investigated in This Exploration

\begin{tabular}{|c|c|c|c|c|}
\hline Patient Idx & $\begin{array}{l}\text { Fetal Depth } \\
\quad(\mathrm{cm})\end{array}$ & \multicolumn{3}{|c|}{$\begin{array}{l}\text { Maternal Heart Rate } \\
\text { (bpm) }\end{array}$} \\
\hline Pt1 & 2.0 & \multirow{7}{*}{60} & \multirow{7}{*}{90} & \multirow{7}{*}{120} \\
\hline Pt2 & 2.5 & & & \\
\hline Pt3 & 3.0 & & & \\
\hline Pt4 & 3.5 & & & \\
\hline Pt5 & 4.0 & & & \\
\hline Pt6 & 4.5 & & & \\
\hline Pt7 & 5.0 & & & \\
\hline
\end{tabular}

performance for given patient and optode parameters, and in doing so, determine the operational limits for feasible designs.

\subsection{Exploration Scope}

To evaluate system performance, we vary $p_{i}$ and $x$ within reasonable bounds to provide useful results in a computationally feasible manner.

5.1.1 Range of Patient Characteristics $\left(P_{p o p}\right)$. To investigate the appropriate range of fetal depths and maternal heart rates, we first look at their incidence in the target patient population, namely near-term pregnant mothers. For simplicity, only singleton pregnancies are considered. Studies have reported that the average depth of a fetus is $2.9 \mathrm{~cm}$ with a standard deviation of $\pm 0.8 \mathrm{~cm}$ (minimum: $1.7 \mathrm{~cm}$ ) [7] and the expected values for the MHR are $81 \mathrm{bpm}$ (with a standard deviation of $\pm 3.97 \mathrm{bpm}$ ) during the relevant stage of pregnancy [26]. To provide a wide-set of patient characteristics considered in our exploration, we explore fetal depths ranging from $2-5 \mathrm{~cm}$ and vary the maternal heart rates between $60-120 \mathrm{bpm}$, thus covering a majority ( 93\%) of the values reported in these studies. Since the system performance on a patient with a fetal depth $3.0 \mathrm{~cm}$ will be similar to another patient with $3.1 \mathrm{~cm}$, we explore fetal depths in $0.5 \mathrm{~cm}$ increments. In the same way, we look at the maternal heart rates of $60 \mathrm{bpm}, 90 \mathrm{bpm}$, and $120 \mathrm{bpm}$. These aspects of patient variability can be seen in Table 1 . Note that in this paper we restrict ourselves to exploring only fetal depth and maternal heart rate but acknowledge that the general problem is more complicated, with many other factors of patient variability that are not considered in this investigation.

5.1.2 Range of Feasible Optode Design Parameters (X). In this exploration, we look at the number of detectors on an optode and their relative placement to the light source. Since the fetal signal is extracted using ANC, a minimum of 2 detectors are needed on the optode. As for the upper-bound, we define our feasible solution space to have a maximum of 8 detectors, since a semi-infinite number of detectors would be computationally prohibitive to explore and clinically impractical. As for their SD distances, a brief survey of large monolithic photodiodes found that they often include at least one dimension of $1 \mathrm{~cm}$. Since the mid-point is used to define the SD distance, we allow detectors to have different SD distances ranging from $0.5-7.5 \mathrm{~cm}$, in $1 \mathrm{~cm}$ increments. We define individual detectors by their SD distance, and assign identifiers shown in Figure 3 which are used to describe individual optode designs. We denote $x_{\max }$ as the design that uses all 8 possible detectors, and represents the solution that captures the widest range of fetal depths, but also uses the most detectors. Since it is computationally-prohibitive 


\begin{tabular}{|c|c|}
\hline $\begin{array}{c}\text { Detector } \\
\underline{\text { Idx }}\end{array}$ & $\begin{array}{c}\text { SD Dist. } \\
\text { (cm) }\end{array}$ \\
\hline D1 & 0.5 \\
\hline D2 & 1.5 \\
\hline D3 & 2.5 \\
\hline D4 & 3.5 \\
\hline D5 & 4.5 \\
\hline D6 & 5.5 \\
\hline D7 & 6.5 \\
\hline D8 & 7.5 \\
\hline
\end{tabular}

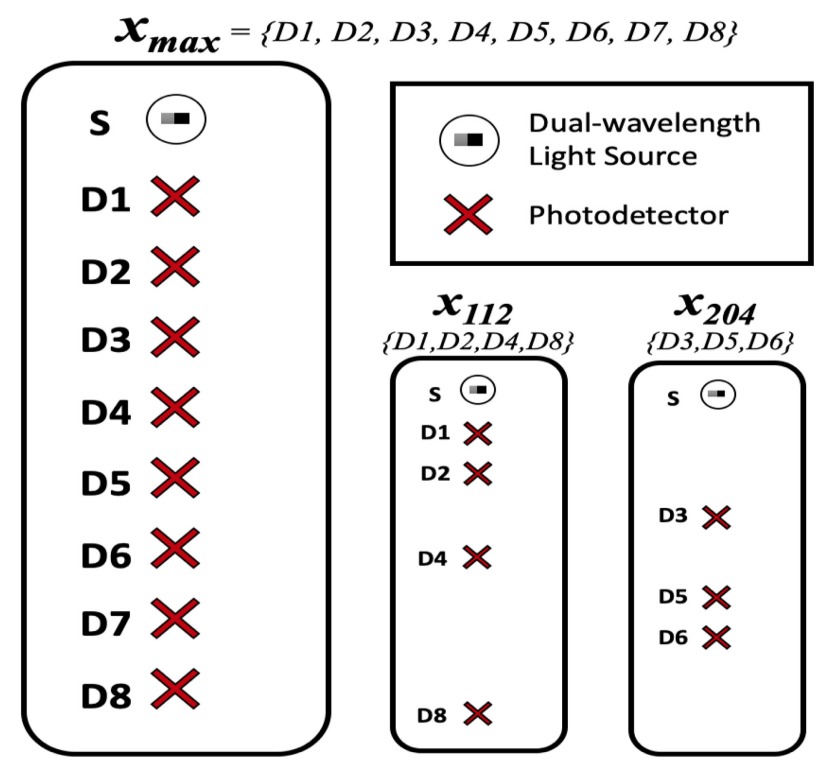

Fig. 3. Detector indexes and their SD distances are shown on the left, and several examples of optode designs on the right.

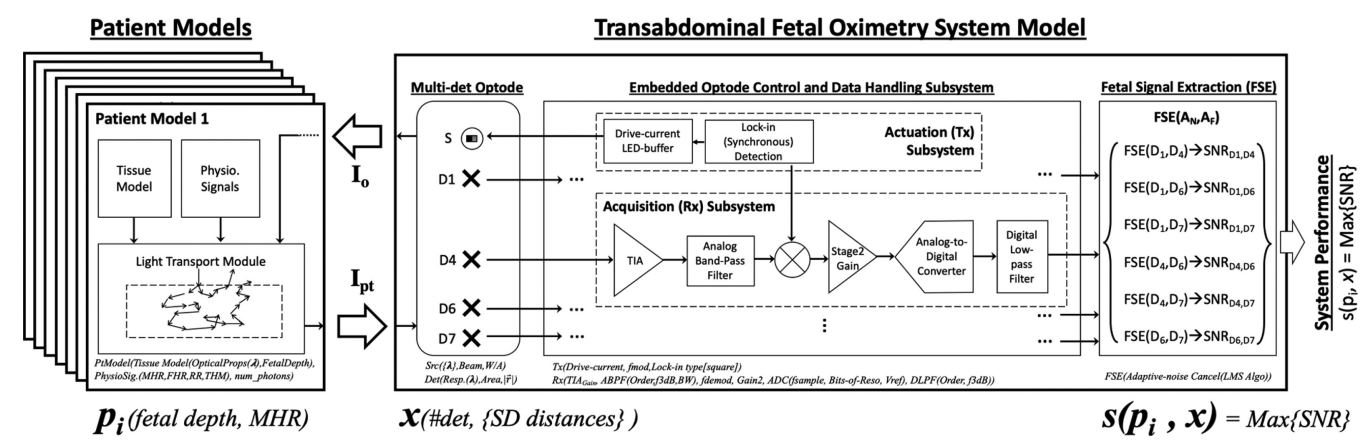

Fig. 4. A high-level view of the interactions that occur within our patient and system models.

to identify the theoretical-maximum population-performance value, this optode provides an empirical-maximum for the population-performance value $\left(S_{p o p}\left(x_{\max }\right)\right)$ achievable.

\subsection{Modeling the Patient and System}

To help with the exploration, we developed patient and system models that encapsulate the exploration parameters and provides a way to evaluate their interaction. A high-level overview of their interactions can be seen in Figure 4.

5.2.1 Patient Model $\left(p_{i}\right)$. Our patient models can be broken up into patient variability submodules and a light-transport module that models photon propagation through tissue to generate the temporal and spatial distribution of the light intensity signal coming from the patient.

Fetal Depth. A key characteristic of patient variability seen in TFO are the differences in fetal depth between pregnant mothers. To capture this variability, we generated tissue models specific to each patient that represents the different tissue types typically seen by a TFO system. In particular, 
Table 2. Optical Properties of Tissue Model

\begin{tabular}{|c|c|c|c|c|c|c|c|c|}
\hline \multirow{2}{*}{ Tissue Layer } & \multicolumn{2}{|c|}{$\mu_{a}\left(\mathrm{~cm}^{-1}\right)$} & \multicolumn{2}{|c|}{$\mu_{s}^{\prime}\left(\mathrm{cm}^{-1}\right)$} & \multirow{2}{*}{ g (unitless) } & \multirow{2}{*}{$\mathrm{n}$ (unitless) } & \multirow{2}{*}{$\mathrm{z}(\mathrm{cm})$} & \multirow{2}{*}{ Ref. } \\
\hline & $\lambda_{735 \mathrm{~nm}}$ & $\lambda_{850 \mathrm{~nm}}$ & $\lambda_{735 \mathrm{~nm}}$ & $\lambda_{850 \mathrm{~nm}}$ & & & & \\
\hline Maternal dermal & 0.170 & 0.125 & 23.0 & 17.7 & 0.9 & 1.4 & 0.15 & [27] \\
\hline Maternal subderm & 0.085 & 0.088 & 12.0 & 11.1 & 0.9 & 1.4 & $0.9-3.9$ & [27] \\
\hline Maternal uterus & 0.160 & 0.100 & 10.8 & 8.15 & 0.9 & 1.4 & 0.85 & {$[24]$} \\
\hline Amniotic fluid & 0.025 & 0.042 & 0.1 & 0.1 & 0.9 & 1.334 & 0.1 & [23] \\
\hline Fetal scalp & 0.157 & 0.157 & 6.81 & 6.23 & 0.9 & 1.3 & 0.2 & [15] \\
\hline Fetal arterial & 0.175 & 0.155 & 35.0 & 30.0 & 0.9 & 1.3 & 0.1 & [35] \\
\hline Fetal skull & 0.210 & 0.215 & 10.9 & 9.1 & 0.9 & 1.3 & 0.15 & {$[15,16]$} \\
\hline Fetal brain & 0.187 & 0.132 & 12.2 & 9.8 & 0.9 & 1.3 & 100 & {$[4,16]$} \\
\hline
\end{tabular}

the tissue model includes seven, semi-infinite layers of tissue, namely the mother's dermal, subdermal and uterine layers, a layer of amniotic fluid, and the fetal scalp, arterial, skull, and brain tissues. The optical properties ${ }^{4}$ and thickness for each tissue type are shown in Table 2 and were characterized in the literature using a combination of in vivo and ex vivo samples. To account for the different fetal depths seen in our patient population, we vary the thickness of the mother's subdermal tissue layer, which is the most variable tissue at the abdomen and is comprised of mostly lipids or fat [16].

Maternal Heart Rate. Another key characteristic of patient variability seen in TFO are changes in the tissue content, caused by physiological systems in the body. In this submodule, we consider several temporally recurring signals that can interfere with extracting the fetal information, namely maternal heart rate (MHR), fetal heart rate (FHR), respiration rate (RR), and Mayer waves (THM), and model them by modulating the light intensity at respective rates to consider their effects on system performance. As previously mentioned, we varied the MHR (60 bpm, $90 \mathrm{bpm}$, and $120 \mathrm{bpm}$ ) and kept the other signals at a steady, but typical, level to focus the scope of this investigation. In particular, they are FHR (168 bpm), RR $(0.5 \mathrm{~Hz})$, and THM $(0.1 \mathrm{~Hz})$.

Light Transport Model. To accurately model the stochastic process of light transport through highly-scattering tissues, we utilize a well-verified Monte Carlo algorithm [30] that injects photon packets into the multi-layered tissue model and follows their propagation till it escapes at the tissue surface or is extinguished due to absorption. Briefly, the algorithm works by specifying the wavelength, initial position, and direction of a photon packet. A propagation length is randomly sampled from an exponential distribution generated using the optical properties of the current tissue a photon is traversing. After considering the probabilistic effects of light absorption, a scattering direction is calculated using the Henyey-Greenstein scattering phase function (while considering Fresnel's equations at tissue boundaries), and the process is repeated until all photons have either exited the tissue or are absorbed. We modified the original source-code to also record the tissue layers a photon traverses and report on the number of photons exiting the tissue at the surface. This allows us to characterize the fetal signal sensitivity across the tissue surface and apply temporal variations in the signal caused by physiological systems according to the tissues each photon traverses. A total of 540-million photons were simulated per wavelength across the patient population. For context, simulating the light propagation of 100-million photons took $13.5 \mathrm{hrs}$ to complete on a computer using an Intel i7 $3 \mathrm{GHz}$ processor. While computationally expensive, this

\footnotetext{
${ }^{4} \mathrm{~A}$ description of the optical properties for biological tissues can be found in [16].
} 
large amount was necessary to accurately model the light-tissue response with our system, which requires the signal to converge at far SD distances.

5.2.2 System Model (x). Our system model is comprised of three submodules, namely a multidetector optode, an embedded optode control and data handling system, and the fetal signal recovery algorithm. Since this work is focused on exploring the optode design, we discuss the multidetector optode's design variables in more detail and briefly describe the other modules and their relevance to system performance.

Multi-detector Optode. In our model, the multi-detector optode is represented as a combination of emitters and detectors. Emitters generate photons which traverse the tissue, and are represented by their peak wavelength, radiative power, and beam profiles. Since oximetry requires two wavelengths of light to be used, we modeled a dual-wavelength, high-power LED with peak wavelengths at $735 \mathrm{~nm}$ and $850 \mathrm{~nm}$, which were shown in previous work to sufficiently propagate to the deeper tissues [9]. We set these high-power LEDs to output $600 \mathrm{~mW}$ of radiative power with a gaussian beam profile. These parameters were sent to the patient model, which models the light-tissue interaction, and returns the spatial- and temporally- varying light intensity signal that interacts with our photodetectors.

Photodetectors are sensors that translate incident light into an electrical signal, and are represented by their responsivity, active area, and SD distance. Responsivity (Amps per Watt or A/W) represents the amount of electrical current or photocurrent $(\mathrm{A})$ that is produced when a particular wavelength of radiative power (W) interacts with its photosensitive active area $\left(\mathrm{cm}^{2}\right)$. The SD distance defines the spatial coordinates of the detector on the optode and is used to determine the level of light intensity hitting its active area. To capture enough light that contains fetal information, we represented the photodetectors as monolithic Si-photodiodes with a large active area $\left(3 \mathrm{~cm}^{2}\right)$ with typical responsivity levels of $0.5 \mathrm{~A} / \mathrm{W}$ and $0.6 \mathrm{~A} / \mathrm{W}$ for the wavelengths $735 \mathrm{~nm}$ and $850 \mathrm{~nm}$, respectively. We varied the number of detectors and SD distances to create different optode designs. The photocurrent generated by each detector was then transferred to the optode control module.

Embedded Optode Control and Data Handling System. Our embedded optode control and data acquisition system helps model the translation of the analog photocurrent into a digitally sampled signal for processing by our fetal signal recovery algorithm. An overview of the different components and their interactions can be seen in Figure 4. Briefly, the current generated at each of the detectors goes through an acquisition ( $\mathrm{Rx}$ ) pipeline which includes a transimpedance amplifier (TIA) to translate the current signal to a voltage, an analog band-pass filter with center point at the lock-in frequency, a demodulator to shift the synchronous signal back to base-band, a secondstage gain circuit, and an analog-to-digital converter to digitize the signal before being processed by the fetal signal extraction algorithm.

Fetal Signal Extraction Algorithm. To evaluate system performance, we must extract the fetal signal using measurements from the various combinations of available detectors on the optode (see Section 2.3.2). This is done through adaptive noise cancellation using the Least-Mean Squares (LMS) algorithm, which attempts to extract the fetal signal for each near-far detector combination. In brief, the LMS algorithm uses gradient descent to iteratively optimize an adaptive filter that identifies the fetal signal using the mean-squared error as its cost function. Since different optodes can have a different number of near-far detector combinations, we use the maximum SNR calculated on an optode to objectively compare the system performance of different designs. 

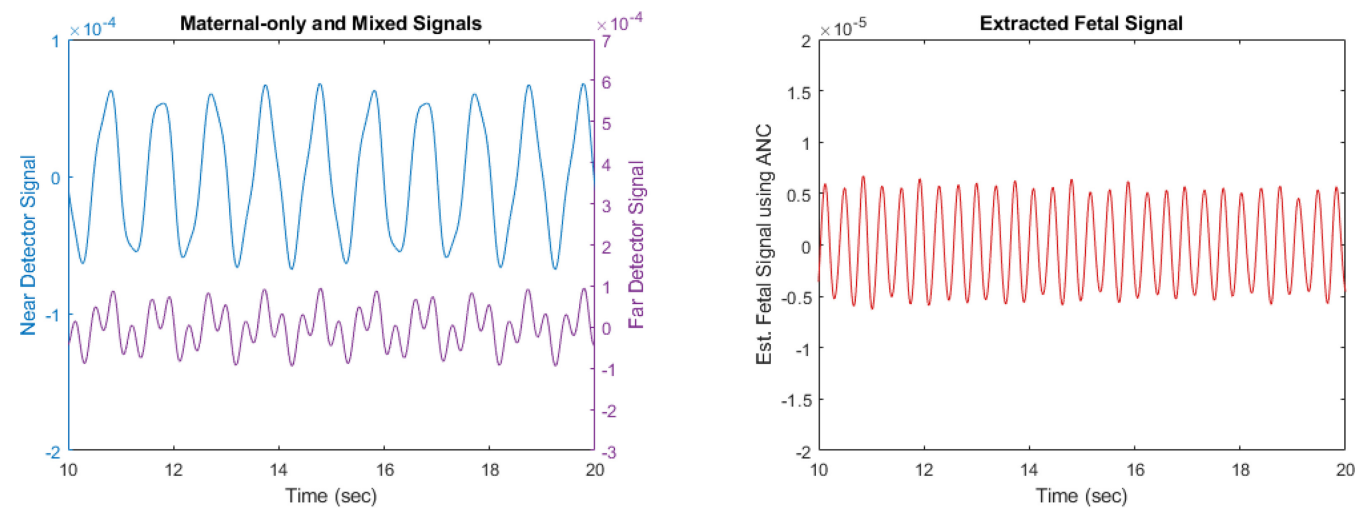

Fig. 5. An example of the signals used to evaluate system performance. Signals from the near-far detectors (D2-D5) are on the left and the extracted fetal signal on the right.

5.2.3 Modeling Patient-System Interaction. We first generated all possible optode designs in the feasible set of solutions, and simulated their interaction with each patient model for a measurement window of 70 seconds using the Simulink framework (MathWorks, Inc.). We allowed the measured signals to converge in the first 10 seconds (which was sufficient time to allow the response of the analog/digital filters to settle) and used the remaining measurements to extract the fetal signal and evaluate the performance for each optode design $(x)$ and patient characteristic $\left(p_{i}\right)$. This process was performed for both wavelengths of light, namely $735 \mathrm{~nm}$ and $850 \mathrm{~nm}$. An example of the measurements taken and the signal extraction can be seen in Figure 5.

\subsection{Identifying a Population-conscious Design}

After characterizing the optode design space, we focus on identifying the Pareto-optimal points that improves the performance for a majority of the patients and minimizes hardware complexity. The first step in our proposed approach involves generating the population-performance metric $\left(S_{\text {pop }}(x)\right)$ using a weighted-value of each patient-specific performance objective $\left(s_{i}(x)\right)$. Afterwards, $S_{\text {pop }}(x)$ is optimized while the number of detectors $(k(x))$ is constrained within user-specified bounds.

5.3.1 Generating the Population-Performance Metric $\left(S_{\text {pop }}(x)\right)$. In our proposed method, we create a population-performance metric $\left(S_{p o p}(x)\right)$ based-on the weighted-summation of each patient's system performance $\left(s_{i}(x)\right)$ using the population-distribution as its weighting function, namely $S_{\text {pop }}(x)=\sum_{i}^{N} w_{i} * s_{i}(x)$. This sets the relative importance of each patient-specific performance objective with respect to the incidence of their particular characteristics in the population. Since TFO measurements are particularly sensitive to changes in fetal depth, we focus on capturing the effects of fetal depth variations across the patient population. We do this by using a normal distribution with a mean of $2.9 \mathrm{~cm}$ fetal depth and variance of $0.64 \mathrm{~cm}^{2}$ [7] as the weighting function to generate each patient-specific weight $\left(w_{i}\right)$. These values are shown in Figure 6 and used for corresponding patients. The sum of all patient's weighted-objective functions were then calculated as $S_{p o p}(x)$, as previously described in Equation (9).

5.3.2 Constraining the Development Costs $(k(x))$. After generating the population-performance metric, we identify the Pareto-optimal design points by optimizing for $S_{p o p}(x)$ while constraining the development costs $k(x)$, as shown in Equation (9). Since we are focused on minimizing $k(x)$, we set the initial upper-bound $\varepsilon$ to be the ideal (minimum) number of detectors (i.e. 2 detectors) 


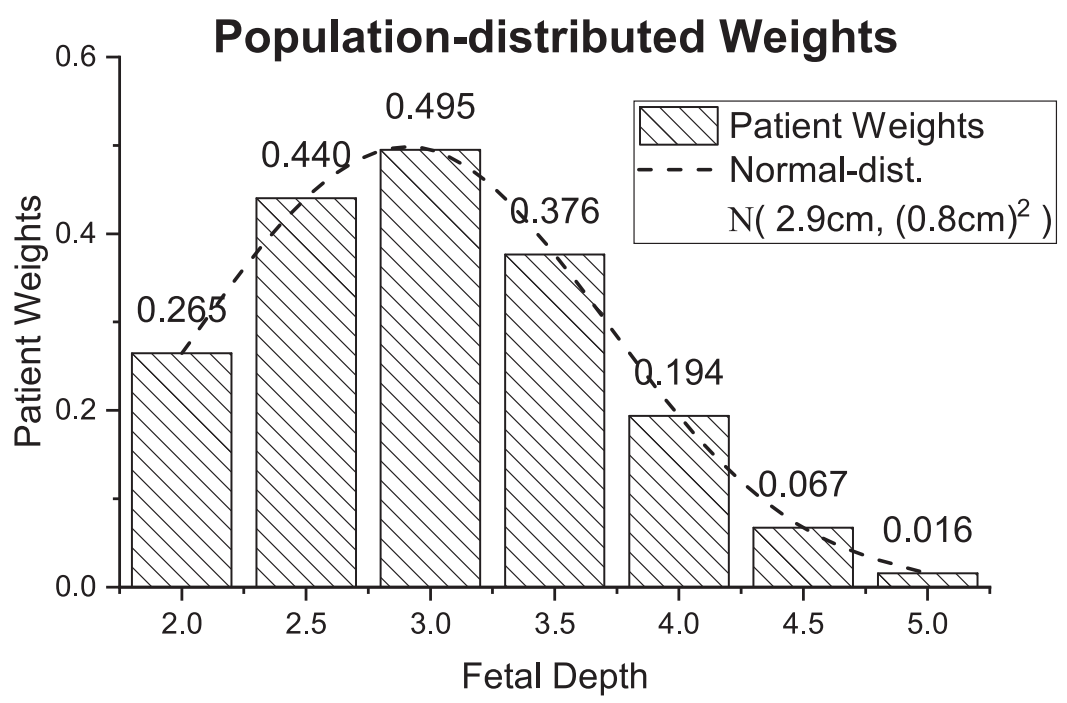

Fig. 6. Patient-specific weights used to generate the Population-performance metric in the proposed approach. These weights set the relative importance of each performance objective with respect to the incidence of their patient characteristics within the population.

which represented the most stringent case. After optimizing for $S_{p o p}$, this bound was iteratively relaxed (increased) to identify designs that may provide a better population-performance but use more detectors. To select a single preferred-solution, we looked at the differences in the populationperformance for each additional detector and searched for the point at which performance benefits had diminishing returns (i.e. $\leq 2 \mathrm{~dB}$ improvement per additional detector).

\section{RESULTS AND DISCUSSION}

\subsection{Characterizing the Optode Design Space}

The system performance was characterized for a total of 247 different optode designs over 7 different fetal depths and 3 different MHRs using 2 different wavelengths of light. To show the highdimensionality of the explored problem, a randomly selected sample of optode designs $(x)$ and their objective functions $\left(s_{i}(x)\right.$ and $\left.k(x)\right)$ are shown in Figure 7.

To highlight the effects that patient variability has on system performance, we use the results from $x_{\max }$ which captures the widest range of fetal depths among the feasible solutions. The effect of fetal depth is shown in Figure 8(A). The performance shown at each fetal depth was averaged over MHR and shown for both wavelengths investigated. Similarly, Figure 8(B) displays the change in performance with respect to maternal heart rate, which was averaged over fetal depth. As expected, these results show that the fundamental ability to detect the fetal signal decreases as the fetus gets deeper, likely due to the fact that the increased distance causes less photons to traverse the fetal tissue and make it back to the surface for detection. In addition, these results also show that the operational limit for the system are patients with fetal depths of $5 \mathrm{~cm}$ using $735 \mathrm{~nm}$ light. While this represents only $\sim 1.6 \%$ of the population, a variety of unexplored parameters could be investigated to improve this, such as using more sensitive optical sensors or using a higher radiative power. On the other hand, the effect that maternal heart rate has on the signal is less concerning since the performance across the population is well above unity $(0 \mathrm{~dB})$ and supports the use of our fetal signal extraction technique. Since the design optimization primarily focuses on addressing 


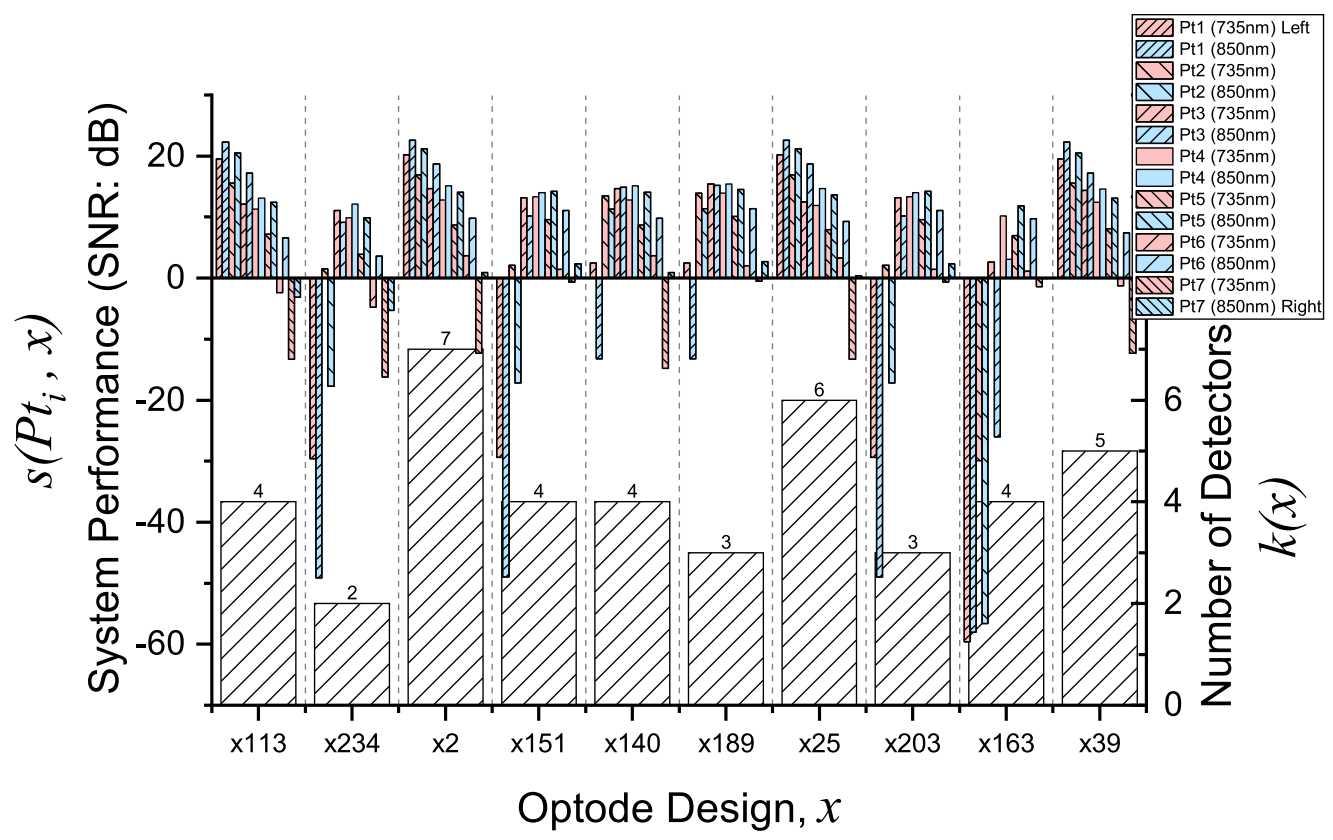

Fig. 7. Objective functions $\left(s_{i}(x)\right.$ and $\left.k(x)\right)$ for a random selection of optode designs $(\mathrm{x})$. These graphs show the high-dimensionality of performance values considered for each optode design.
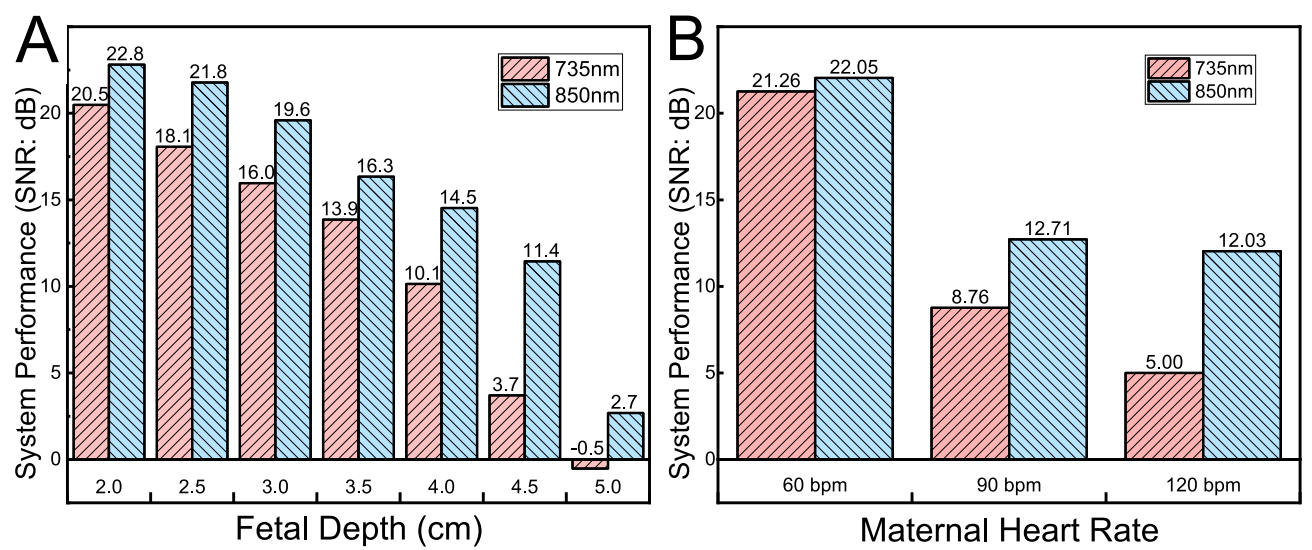

Fig. 8. Patient variability effects on TFO System Performance using $x_{\max }$. The system performance values shown at each fetal depth (A) was averaged over maternal heart rate (MHR). Similarly, values shown at each MHR (B) was averaged over each fetal depth.

fetal depth variations, we use the mean performance across MHR to compare different optodes using the proposed method.

Figure 9 displays a heat-map of the SNR at individual near-far detector combinations on the $x_{\max }$ optode, and shows how the optimal near-far detector location changes as the fetal depth increases. Importantly, this image also highlights how the inclusion (or exclusion) of particular SD distances can impact the performance captured by an optode on different patients. 

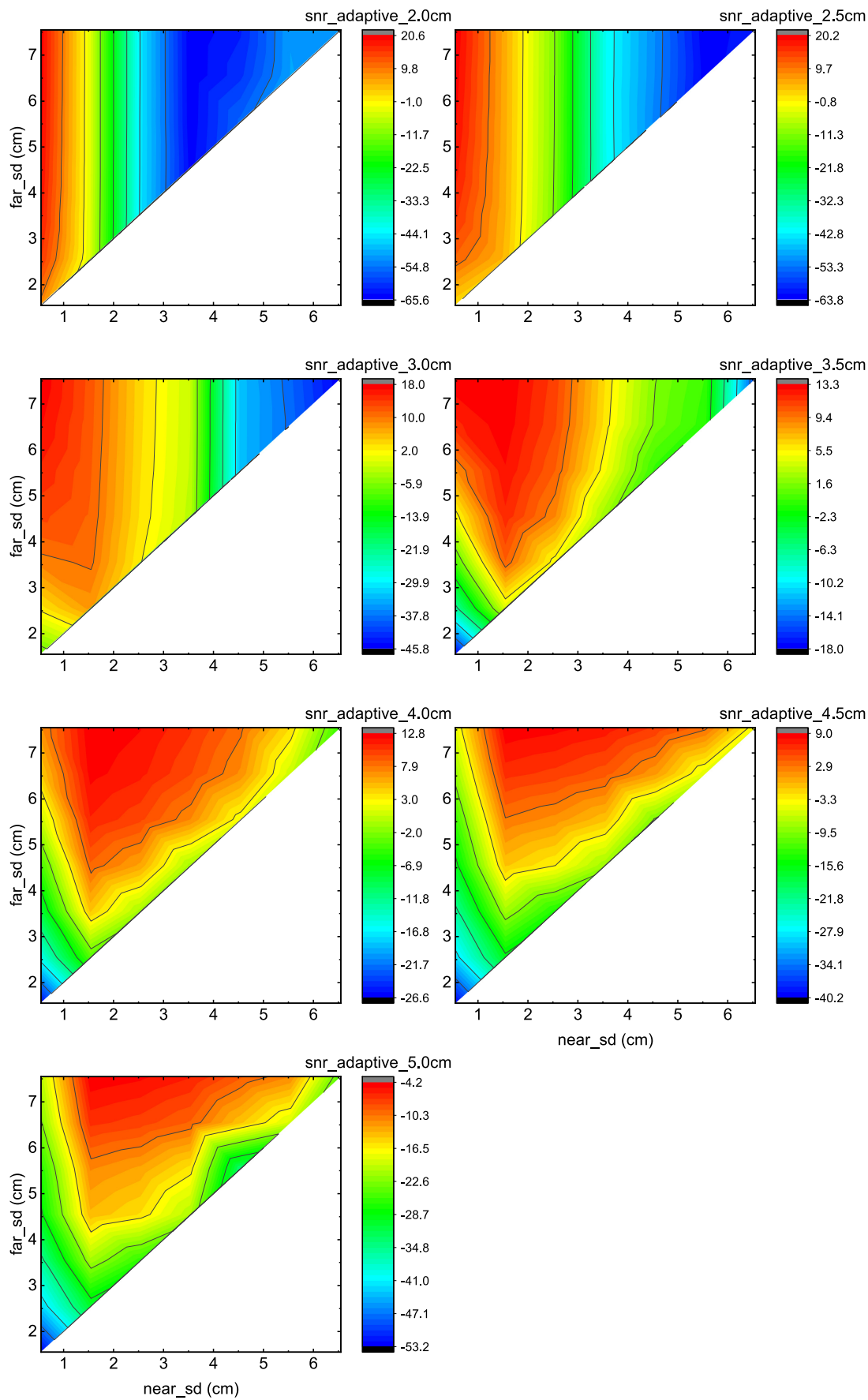

Fig. 9. A heat map of the signal-to-noise ratio $(\mathrm{dB})$ for different patients at each near-far detector combination on $x_{\max }$. As shown, the optimal near-far detector location changes as the fetal depth increases. In addition, the inclusion/exclusion of particular SD distances affects the overall performance seen across patients. 


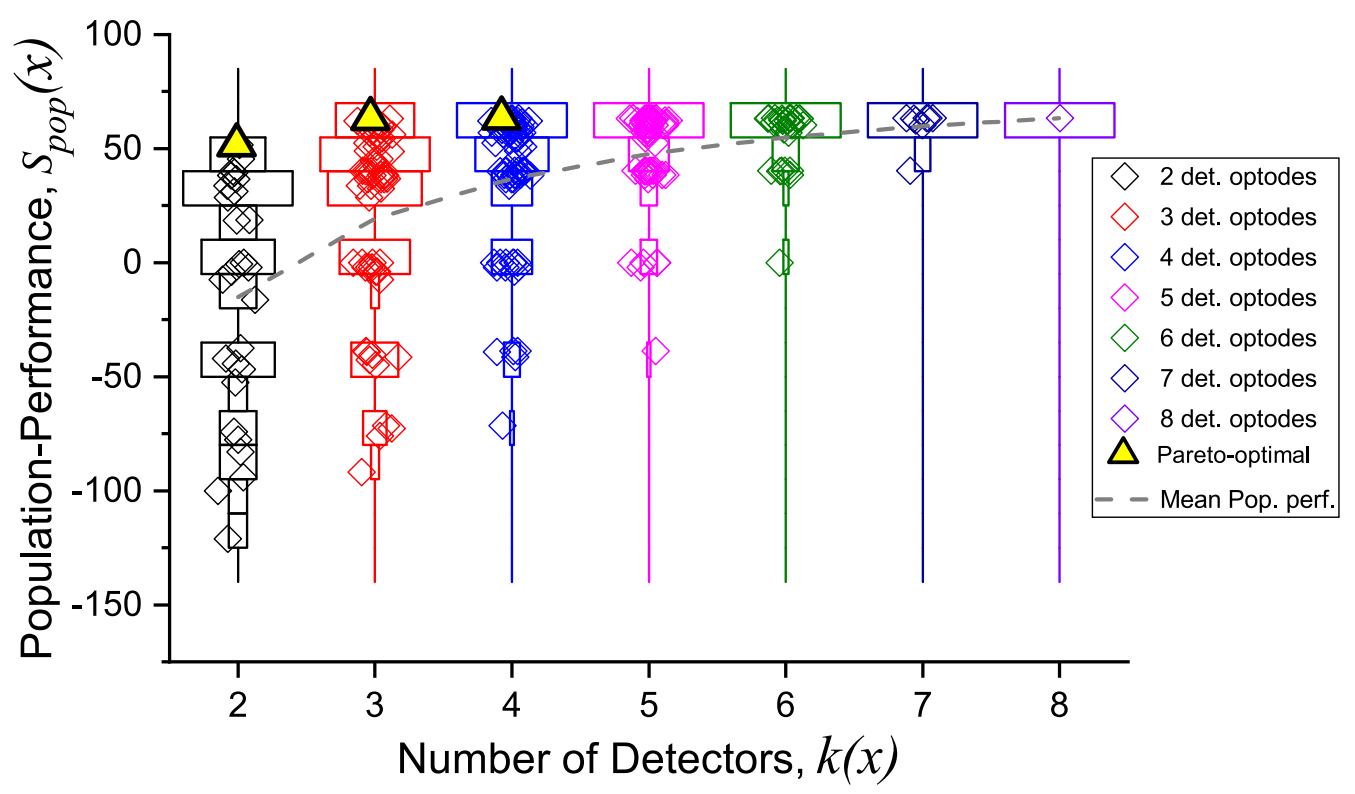

Fig. 10. Population-performance for all optode designs with respect to number of detectors.

Table 3. Population-conscious Optode Designs Identified using the Proposed Method

\begin{tabular}{cccc}
\hline Optode Design & Detectors & No. of Detectors & Population-Performance \\
\hline$x_{\max }$ & (D1, D2, D3, D4, D5, D6, D7, D8) & 8 & $63.30 \mathrm{~dB}$ \\
$x_{4}$ & (D1, D2, D3, D4, D5, D7, D8) & 7 & $63.30 \mathrm{~dB}$ \\
$x_{15}$ & (D1, D2, D3, D4, D7, D8) & 6 & $63.30 \mathrm{~dB}$ \\
$x_{47}$ & (D1, D2, D3, D7, D8) & 5 & $63.30 \mathrm{~dB}$ \\
$x_{108}{ }^{*}$ & (D1, D2, D7, D8) & 4 & $63.30 \mathrm{~dB}$ \\
$\mathbf{x}_{169}{ }^{*}$ & "(D1, D2, D8) & 3 & $\mathbf{6 3 . 1 9} \mathrm{dB}$ \\
$x_{226}{ }^{*}$ & (D1, D8) & 2 & $51.62 \mathrm{~dB}$ \\
\hline
\end{tabular}

*Pareto-optimal designs.

\subsection{Pareto-optimal Solutions}

Population-performance metrics were generated for all 247 optodes using the proposed method and are shown with respect to the number of detectors in Figure 10. The optode designs that maximized the population-performance were identified for different amounts of detectors, and are shown in Table 3. The Pareto-optimal solutions $\left(S_{p o p}(x)\right)$ are marked with asterisks. These results show that the population performance achieved using the maximal 8 detectors $\left(x_{\max }\right)$ can also be achieved by carefully placing 4 detectors at appropriate locations on the optode according to $x_{108}$. However, since the population performance benefit between 3 and 4 detectors is only $0.11 \mathrm{~dB}$, we select $x_{169}$ as the preferred Pareto-optimal optode design. When compared with the optode design used in the current state of the art [36] $\left(x_{156}=(D 3, D 5, D 6, D 8)\right)$, we see that the proposed approach selects a design that provides a much better population-performance $\left(S_{\text {pop }}\left(x_{156}\right)=-0.13 \mathrm{~dB}\right)$. In particular, these results suggest that the optode design $x_{169}$ is able to address issues related to patient variability using only 3 detectors. 

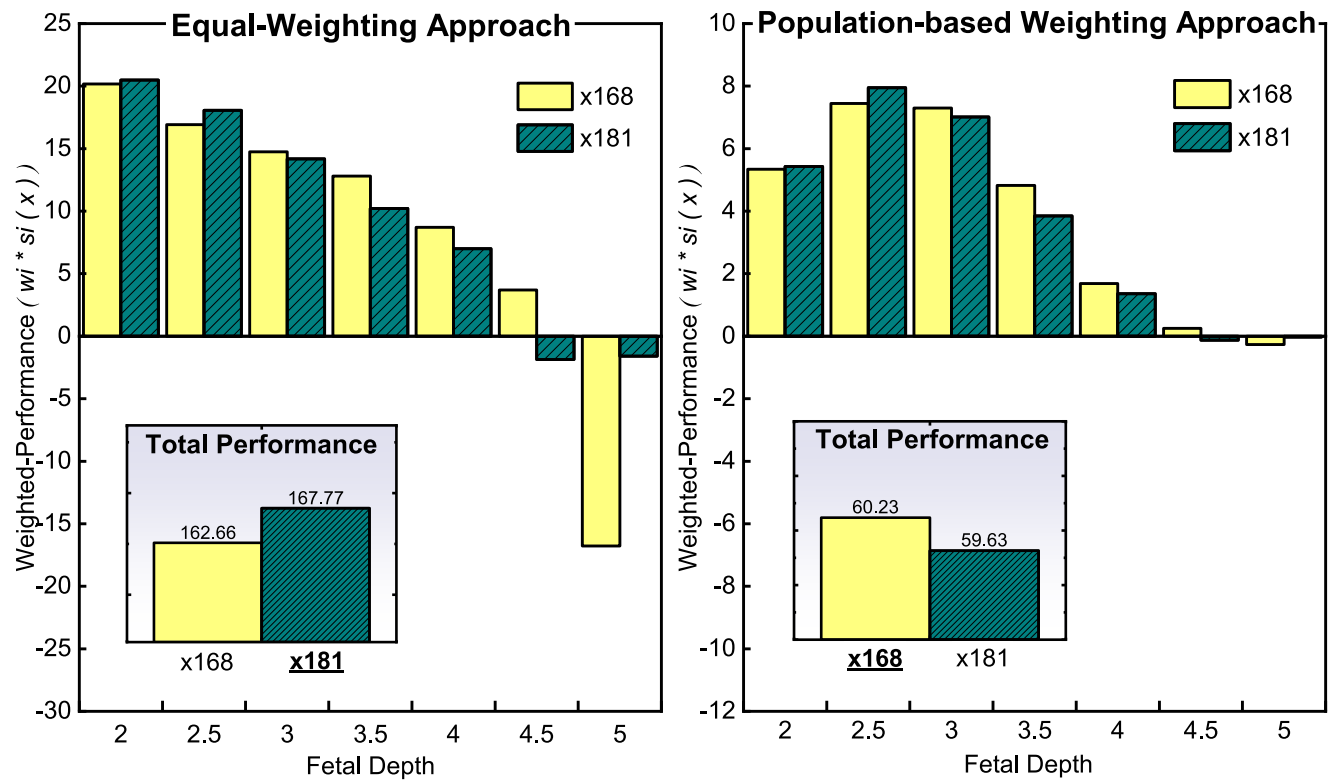

Fig. 11. A comparison of Equal weighting vs Population-based weighting methods for designs $x_{168}$ and $x_{181}$. For visual clarity, only $735 \mathrm{~nm}$ results are displayed. Total performance is the sum of each patient specific (fetal depth) performance. As shown, the poor performance for fetal depths of $5 \mathrm{~cm}$ is mitigated in the proposed population-based weighting method, by considering the percentage of the population that has fetal depths of $5 \mathrm{~cm}(\sim 1.6 \%)$, which allows for the selection of an optode that provides better performance for the population.

To highlight the beneficial effect that population-based weighting has in the proposed approach, we consider the case when only two of the explored optode designs are available $\left(x_{168} \text { and } x_{181}\right)^{5}$ and compare the results when each patient performance is treated equally, namely $w_{i}=1$. Each design's patient-specific weighted-performance $\left(w_{i} * s_{i}(x)\right)$ is shown in Figure 11. As seen in the equal-weighting method, although $x_{168}$ performs similarly as $x_{181}$ across most patients, it performs terribly on fetal depths of $5 \mathrm{~cm}$, and results with $x_{181}$ being selected as the preferred design. However, only $\sim 1.6 \%$ of the population have these large fetal depths. The proposed population-based weighting accounts for this by appropriately scaling the impact each patient's performance has to the overall population, and instead suggests $x_{168}$ as the preferred design. As we can see for the more common fetal depths, $x_{168}$ provides better performance than $x_{181}$, which supports the efficacy of the proposed method to select designs that maximizes the performance for the population.

\subsection{Preliminary In Vivo Results}

To establish in vivo feasibility, we developed a non-invasive fetal oximetry system and captured measurements on a pregnant sheep animal model ${ }^{6}$ as a step towards working with pregnant women.

6.3.1 Experimental Setup. Near-term pregnant ewes, that were scheduled for a planned Csection, were utilized for this study. Prior to data acquisition, anesthesia was induced and

\footnotetext{
${ }^{5} x_{168}=(D 1, D 2, D 7)$ and $x_{181}=(D 1, D 5, D 8)$.

${ }^{6}$ Pregnant sheep are often utilized as an animal model for human pregnancy due to their similarities with human physiology.
} 

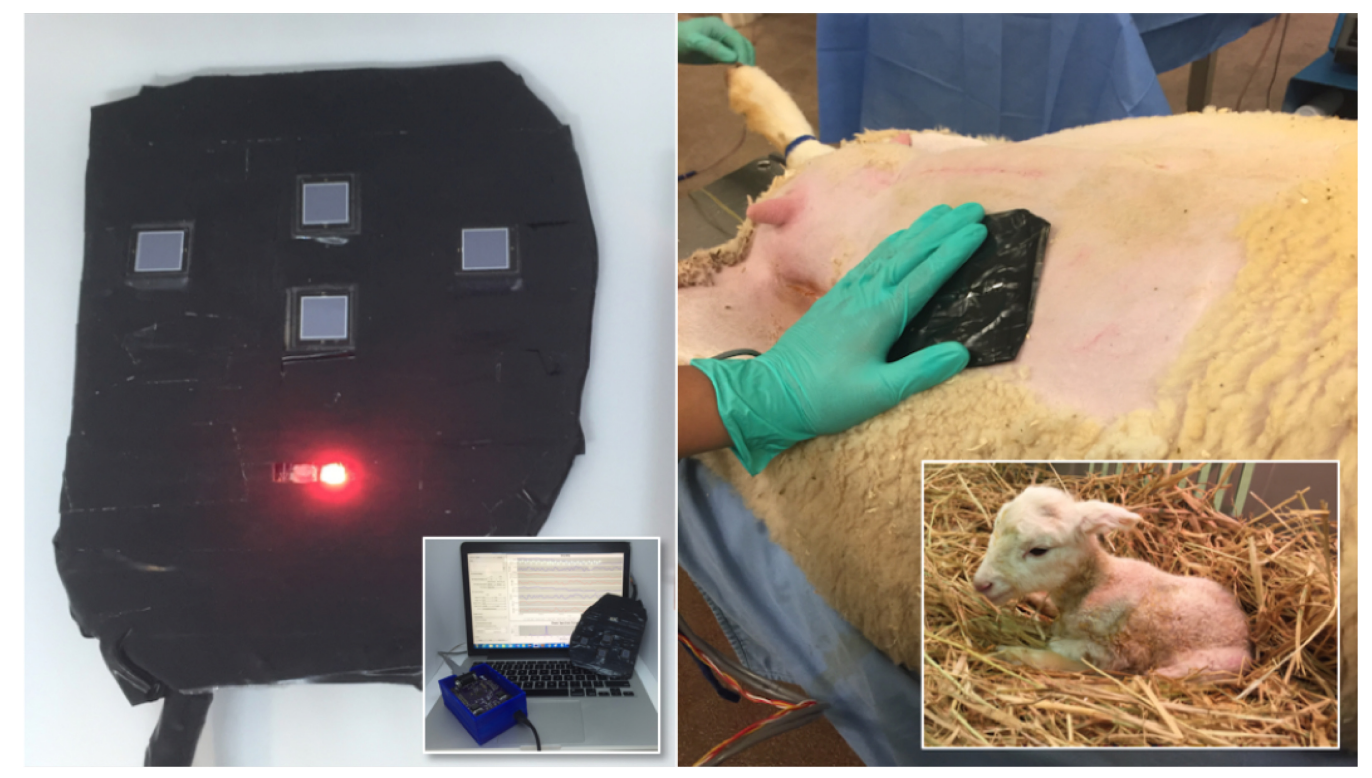

Fig. 12. Pictures of the multi-detector optode (left) used with our system (inset) to capture signals on pregnant sheep (right).

abdominal region was shaved as part of the preparation for surgery. A lingual pulse oximeter captured the maternal heart rate during the measurements using our system. After palpating for the fetal location, our TFO system's optode was placed directly above the fetus and several minutes of data was captured. Afterwards, the fetal lamb was delivered via C-section, allowed to recover, and confirmed alive. All procedures described here were approved by an Institutional Animal Care and Use Committee.

To capture the signals on the pregnant sheep, we developed a TFO system that consisted of a dual-detector optode, embedded control system, and custom software (written in Java) that captured and displayed measurements in real-time, and is shown in Figure 12. Our dual-detector optode contained a pair of near and far detectors (Si-photodiodes) with SD distances of $4 \mathrm{~cm}$ and $7 \mathrm{~cm}$ respectively and total active areas of $1 \mathrm{~cm}^{2}$ and $3 \mathrm{~cm}^{2}$ respectively. To extract the fetal signal, we used adaptive noise cancellation with the LMS algorithm where the measurements at the near and far detectors provide the noise-reference and noise-corrupted-signal, respectively.

6.3.2 Experimental Results. Measurements captured on pregnant sheep using our system can be seen in Figure 13. The signals at the near and far detectors can be seen in the top and middle rows respectively, and the extracted fetal signal is shown in the bottom row. To visualize the signal over the entire measurement period, we display each of their spectrograms ${ }^{7}$ in Figure 13(B). Yellow lines in the spectrogram indicate strong frequency components of the measured signal (and their harmonics) and are annotated with the physiological source. As shown, the maternal signal $(\sim 1.25 \mathrm{~Hz})$ can be seen in the spectrograms of both near and far detectors. After extracting the fetal signal from the mixed signal measurements at the far detector, we can see that the maternal noise has been reduced and the fetal signal can be seen at $(\sim 3.7 \mathrm{~Hz})$. Due to limitations of the experimental protocol, the fetal heart rate was not taken at the same time as the TFO measurements. However, discussions with clinicians and surgical staff confirmed that the only physiological

\footnotetext{
${ }^{7}$ A spectrogram displays the changes in the frequency content of a signal over time.
} 

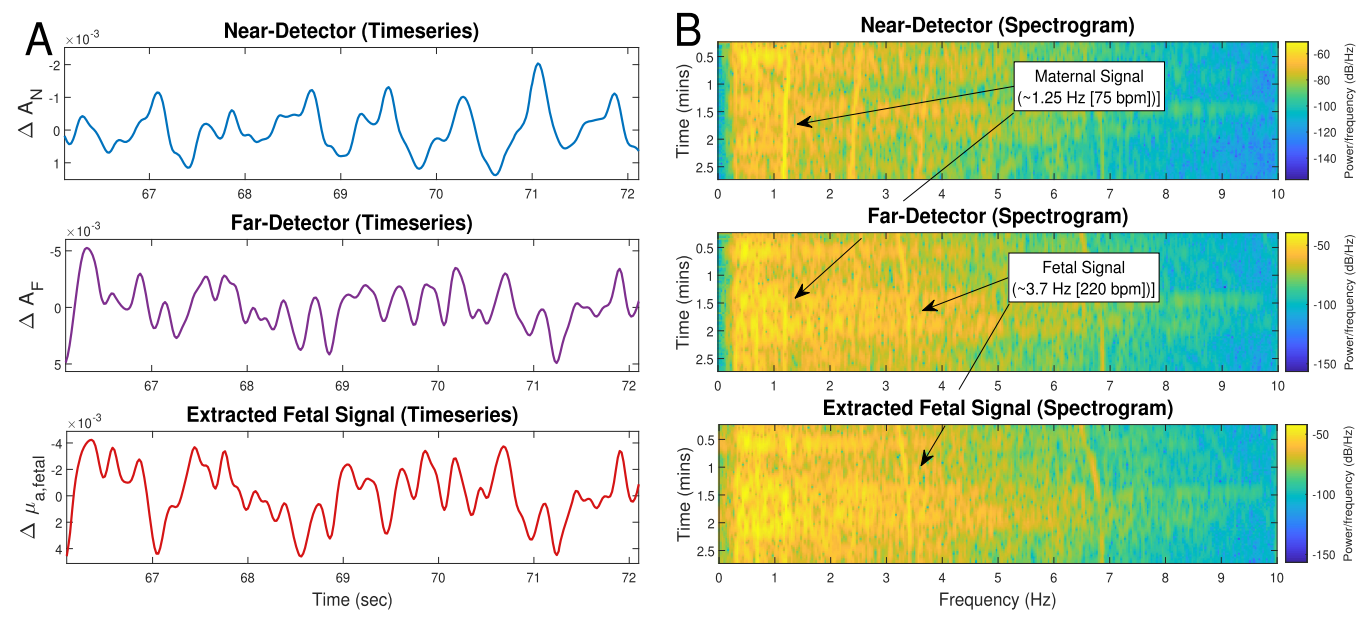

Fig. 13. Measurements captured at the near (top row) and far detectors (middle row) on the pregnant sheep are used to extract the fetal signal (bottom row), and are displayed in the time-domain (A) and frequencydomain (B). Yellow lines in the spectrogram indicate strong frequency components of the measured signal (and their harmonics) and are annotated with the physiological source.

signal present around $\sim 3.7 \mathrm{~Hz}$ is the fetal heart rate, improving our confidence that the fetal signal was captured. These results show that our system is able to capture in vivo measurements of the fetal signal on near-term pregnant sheep and highlight its feasibility towards future experiments involving pregnant women.

\section{RELATED WORKS}

A large multi-center clinical study found that transcervical fetal pulse oximetry provided physicians with a better understanding of fetal well-being [12]. This semi-invasive method estimated fetal $\mathrm{SpO}_{2}$ by placing a pulse oximeter through the birth canal and onto fetal tissues. As such, several research groups have been working on the development of a fully non-invasive system, which is faced with several additional challenges including light attenuation, mixed-signal measurements, and patient variability.

Several studies have used Monte Carlo simulations to model photon transport in non-invasive fetal oximetry to investigate the maternal-fetal signal contribution $[5,9,11,29,36]$, which showed the large influence tissue geometries have on the fetal signal sensitivity. Some were complemented with measurements across optical phantoms (which mimic the optical properties of tissue) [10, 29] and showed the validity of the simulation results using representative physical models. Others have emulated the mixed-signal measurements through a variety of means to investigate various techniques to extract the fetal signal $[10,28,33]$. Furthermore, some have tried to estimate fetal oxygenation using other spectroscopic techniques, namely near-infrared spectroscopy, by characterizing the expected path-length photons take for specific tissue models [22]. This technique does not rely on pulsating arterial tissue, but makes assumptions on (or requires estimations of) the expected path-lengths photons traversing the tissue have and can be drastically different for different fetal depths. In a preliminary study involving pregnant mothers, investigators saw that differences in fetal depth had a large effect on the signal-to-noise ratio, where $\sim 70 \%$ of participants had a SNR $<2$ [36], highlighting the effects that patient variability can have on performance. Addressing these variability issues has been largely unexplored and was the focus of this paper. 


\section{CONCLUSION AND FUTURE WORK}

In this paper, we proposed a methodology to help with the design of a clinically-robust system that addresses patient variability, which is framed as a multi-objective optimization problem. We used the proposed approach to optimize the design of an optode for non-invasive fetal oximetry, and presented both simulated and in vivo measurements captured on pregnant sheep to support the approach. We are currently working on an improved experimental protocol involving pregnant sheep to simultaneously capture the fetal signal via other modalities while using our TFO system to establish ground-truth measurements.

\section{ACKNOWLEDGMENTS}

This material is based upon work supported by the National Science Foundation under Grant No. IIS-1838939. Support is also acknowledged from NIH grants NS094681, EB023591, NS105043, EY028287. One of the authors (D. Fong) would like to acknowledge support from the Floyd and Mary Schwall DY Fellowship in Medical Research. In addition, we would like to thank the members of the UC Davis Surgical Bioengineering Laboratory and Large Animal Surgery Facility staff for their assistance with the in vivo measurements.

\section{REFERENCES}

[1] Z. Alfirevic, D. Devane, G. Gyte, and A. Cuthbert. 2017. Continuous cardiotocography (CTG) as a form of electronic fetal monitoring (EFM) for fetal assessment during labour. Cochrane Database of Systematic Reviews 2 (2017).

[2] Emma L. Barber, Lisbet S. Lundsberg, Kathleen Belanger, Christian M. Pettker, Edmund F. Funai, and Jessica L. Illuzzi. 2011. Indications contributing to the increasing cesarean delivery rate. Obstetrics and Gynecology 118, 1 (July 2011).

[3] Beer. 1852. Bestimmung der absorption des rothen Lichts in farbigen Flüssigkeiten. Annalen der Physik und Chemie (1852).

[4] Frédéric Bevilacqua, Dominique Piguet, Pierre Marquet, Jeffrey D. Gross, Bruce J. Tromberg, and Christian Depeursinge. 1999. In vivo local determination of tissue optical properties: Applications to human brain. Appl. Opt. 38, 22 (Aug 1999).

[5] M. Böttrich, S. Ley, and P. Husar. 2015. Simulation study on the effect of tissue geometries to fluence composition for non-invasive fetal pulse oximetry. In 2015 37th Annual International Conference of the IEEE Engineering in Medicine and Biology Society (EMBC). 5122-5125.

[6] C. R. Cardwell, L. C. Stene, G. Joner, O. Cinek, J. Svensson, M. J. Goldacre, R. C. Parslow, P. Pozzilli, G. Brigis, D. Stoyanov, B. Urbonaite, S. Šipetić, E. Schober, C. Ionescu-Tirgoviste, G. Devoti, C. E. de Beaufort, K. Buschard, and C. C. Patterson. 2008. Caesarean section is associated with an increased risk of childhood-onset type 1 diabetes mellitus: A meta-analysis of observational studies. Diabetologia 51, 5 (May 2008), 726-735.

[7] Paul L. Carson, Jonathan M. Rubin, and Edward H. Chiang. 1989. Fetal depth and ultrasound path lengths through overlying tissues. Ultrasound in Medicine and Biology 15, 7 (1989), 629-639.

[8] D. T. Delpy, M. Cope, P. van der Zee, S. Arridge, S. Wray, and J. Wyatt. 1988. Estimation of optical pathlength through tissue from direct time of flight measurement. Physics in Medicine and Biology 33, 12 (1988).

[9] Daniel Fong, André Knoesen, and Soheil Ghiasi. 2017. Transabdominal fetal pulse oximetry: The case of fetal signal optimization. In 2017 IEEE 19th International Conference on e-Health Networking, Applications and Services (Healthcom).

[10] Daniel D. Fong, André Knoesen, Mohammad Motamedi, Terry O’Neill, and Soheil Ghiasi. 2018. Recovering the fetal signal in transabdominal fetal pulse oximetry. Smart Health 9-10 (2018), 23-36.

[11] K. B. Gan, E. Zahedi, and M. A. Mohd Ali. 2011. Investigation of optical detection strategies for transabdominal fetal heart rate detection using three-layered tissue model and Monte Carlo simulation. Optica Applicata 41, 4 (2011).

[12] Thomas J. Garite, Gary A. Dildy, Helen McNamara, Michael P. Nageotte, Frank H. Boehm, Eric H. Dellinger, Robert A. Knuppel, Richard P. Porreco, Hugh S. Miller, Shiraz Sunderji, Michael W. Varner, and David B. Swedlow. 2000. A multicenter controlled trial of fetal pulse oximetry in the intrapartum management of nonreassuring fetal heart rate patterns. American fournal of Obstetrics and Gynecology 183, 5 (11 2000), 1049-1058.

[13] Yacov Haimes, Leon Lasdon, and David Wismer. 1971. On a bicriterion formulation of the problems of integrated system identification and system optimization. IEEE Transactions on Systems, Man, and Cybernetics SMC-1, 3 (July 1971), 296-297.

[14] Anne Kirkeby Hansen, Kirsten Wisborg, Niels Uldbjerg, and Tine Brink Henriksen. 2008. Risk of respiratory morbidity in term infants delivered by elective caesarean section: Cohort study. BM7 336, 7635 (2008), 85-87. 
[15] Sonoko Ijichi, Takashi Kusaka, Kenichi Isobe, Kensuke Okubo, Kou Kawada, Masanori Namba, Hitoshi Okada, Tomoko Nishida, Tadashi Imai, and Susumu Itoh. 2005. Developmental changes of optical properties in neonates determined by near-infrared time-resolved spectroscopy. Pediatr Res 58, 3 (09 2005), 568-573.

[16] Steven L. Jacques. 2013. Optical properties of biological tissues: A review. Physics in Medicine and Biology 58, 11 (2013).

[17] D. F. Jones, S. K. Mirrazavi, and M. Tamiz. 2002. Multi-objective meta-heuristics: An overview of the current stateof-the-art. European fournal of Operational Research 137, 1 (2002), 1-9.

[18] Johann Heinrich Lambert. 1760. Photometria Sive de Mensura et Gradibus Luminis, Colorum et Umbrae.

[19] Joyce A. Martin, Brady E. Hamilton, Michelle J. K. Osterman, Anne K. Driscoll, and Patrick Drake. 2018. Births: Final data for 2017. National Vital Statistics Reports (2018).

[20] Karin B. Nelson, James M. Dambrosia, Tricia Y. Ting, and Judith K. Grether. 1996. Uncertain value of electronic fetal monitoring in predicting cerebral palsy. New England fournal of Medicine 334, 10 (1996), 613-619.

[21] Thorkild F. Nielsen and Klas-Henry Hökegård. 1984. Cesarean section and intraoperative surgical complications. Acta Obstetricia et Gynecologica Scandinavica 63, 2 (1984), 103-108.

[22] Nirmala Ramanujam, Hong Long, Martha Rode, Iraj Forouzan, Mark Morgan, and Britton Chance. 1999. Antepartum, transabdominal near infrared spectroscopy: Feasibility of measuring photon migration through the fetal head in utero. The fournal of Maternal-Fetal Medicine 8, 6 (1999), 275-288.

[23] Y. L. Narasinga Rao, Vani Isuapalli, and Sai Samyukta Ila. 2015. Amniotic fluid optical density (AFOD) correlates with the lung maturity as well as complete maturity of the fetus - A clinical observational study. International Archives of Integrated Medicine 2, 8 (August 2015).

[24] P. M. Ripley, J. G. Laufer, A. D. Gordon, R. J. Connell, and S. G. Bown. 1999. Near-infrared optical properties of ex vivo human uterus determined by the Monte Carlo inversion technique. Physics in Medicine and Biology 44, 10 (1999), 2451.

[25] Rolf B. Saager and Andrew J. Berger. 2005. Direct characterization and removal of interfering absorption trends in two-layer turbid media. F. Opt. Soc. Am. A 22, 9 (Sep 2005), 1874-1882.

[26] Dan J. Sherman, Eugenia Frenkel, Yaffa Kurzweil, Anna Padua, Shlomo Arieli, and Murat Bahar. 2002. Characteristics of maternal heart rate patterns during labor and delivery. Obstetrics and Gynecology 99, 4 (2002), 542-547.

[27] C. Rebecca Simpson, Matthias Kohl, Matthias Essenpreis, and Mark Cope. 1998. Near-infrared optical properties of ex vivo human skin and subcutaneous tissues measured using the Monte Carlo inversion technique. Physics in Medicine and Biology 43, 9 (1998), 2465.

[28] Norbert Stuban, Masatsugu Niwayama, and Hunor Santha. 2012. Phantom with pulsatile arteries to investigate the influence of blood vessel depth on pulse oximeter signal strength. Sensors (Basel, Switzerland) 12, 1 (2012), 895-904.

[29] Gargi Vishnoi, Andreas H. Hielscher, Nirmala Ramanujam, and Britton Chance. 2000. Photon migration through fetal head in utero using continuous wave, near-infrared spectroscopy: Development and evaluation of experimental and numerical models. Journal of Biomedical Optics 5, 2 (2000), 163-172.

[30] Lihong Wang, Steven L. Jacques, and Liqiong Zheng. 1995. MCML - Monte Carlo modeling of light transport in multi-layered tissues. Computer Methods and Programs in Biomedicine 47, 2 (1995), 131-146.

[31] J. G. Webster. 1997. Design of Pulse Oximeters. Institute of Physics Publishing.

[32] L. Zadeh. 1963. Optimality and non-scalar-valued performance criteria. IEEE Trans. Automat. Control 8, 1 (1963).

[33] Edmond Zahedi and Gan Kok Beng. 2008. Applicability of adaptive noise cancellation to fetal heart rate detection using photoplethysmography. Computers in Biology and Medicine 38, 1 (1 2008), 31-41.

[34] Quan Zhang, Emery N. Brown, and Gary E. Strangman. 2007. Adaptive filtering for global interference cancellation and real-time recovery of evoked brain activity: A Monte Carlo simulation study. F. of Biomedical Optics 12, 4 (2007).

[35] W. G. Zijlstra, A. Buursma, and W. P. Meeuwsen-van der Roest. 1991. Absorption spectra of human fetal and adult oxyhemoglobin, de-oxyhemoglobin, carboxyhemoglobin, and methemoglobin. Clin Chem 37, 9 (Sep 1991), 1633-1638.

[36] Anna Zourabian, Andy Siegel, Britton Chance, Nirmala Ramanujam, Martha Rode, and David A. Boas. 2000. Transabdominal monitoring of fetal arterial blood oxygenation using pulse oximetry. F. of Biomedical Optics 5, 4 (2000).

Received April 2019; revised June 2019; accepted July 2019 